\title{
Defective insulin secretion and increased susceptibility to experimental diabetes are induced by reduced Akt activity in pancreatic islet $\beta$ cells
}

\author{
Ernesto Bernal-Mizrachi, Szabolcs Fatrai, James D. Johnson, Mitsuru Ohsugi, Kenichi Otani, \\ Zhiqiang Han, Kenneth S. Polonsky, and M. Alan Permutt
}

Division of Endocrinology, Metabolism and Lipid Research, Washington University School of Medicine, St. Louis, Missouri, USA.

\begin{abstract}
The insulin and IGF signaling pathways are critical for development and maintenance of pancreatic $\beta$ cell mass and function. The serine-threonine kinase Akt is one of several mediators regulated by these pathways. We have studied the role of Akt in pancreatic $\beta$ cell physiology by generating transgenic mice expressing a kinasedead mutant of this enzyme in $\beta$ cells. Reduction of Akt activity in transgenic animals resulted in impaired glucose tolerance due to defective insulin secretion. The mechanisms involved in dysregulation of secretion in these mice lie at the level of insulin exocytosis and are not the result of abnormalities in glucose signaling or function of voltage-gated $\mathrm{Ca}^{2+}$ channels. Therefore, transgenic mice showed increased susceptibility to developing glucose intolerance and diabetes following fat feeding. These observations suggest that Akt plays a novel and important role in the regulation of distal components of the secretory pathway and that this enzyme represents a therapeutic target for improvement of $\beta$ cell function in diabetes.
\end{abstract}

\section{Introduction}

Type 2 diabetes is a metabolic disease characterized by insulin resistance and defective insulin secretion. The defects that result in diabetes are diverse, but pancreatic $\beta$ cell failure is a major component of this disease and is often associated with decreased $\beta$ cell mass $(1,2)$. Normally, pancreatic $\beta$ cell mass results from a dynamic balance of neogenesis, proliferation, cell size, and apoptosis (3). Experiments performed in insulinoma cells and the abnormalities demonstrated in animal models deficient in insulin receptor, insulin receptor substrate (IRS) proteins, and insulin-like growth factor I (IGFI) receptor suggest that insulin/IGFI/PI3K signaling pathways might mediate peripheral insulin action as well as pancreatic $\beta$ cell function by regulating proliferation, survival, and insulin secretion (4-12).

Receptor tyrosine kinases like the insulin and IGFI receptors can signal through different pathways that include Ras/MAPKs and PI3K. A major target of PI3K is the serine-threonine kinase Akt, the cellular homologue of the viral oncogene v-Akt (13). Three highly conserved isoforms of Akt/protein kinase $\mathrm{B}(\mathrm{PKB})$, the products of separate genes, have now been identified in mammalian cells (14). Recently, experiments in genetically modified animal models have assessed the role of Akt 1 and Akt2 in glucose homeostasis. Akt1deficient mice exhibited impairment in organ growth with normal glucose tolerance and insulin-stimulated glucose disposal $(15,16)$. In contrast, Akt $2 / \mathrm{PKB} \beta$-deficient mice have impaired glucose disposal due to a reduction in insulin-stimulated glucose uptake in

Nonstandard abbreviations used: $\left[\mathrm{Ca}^{2+}\right]_{\mathrm{i}}$, intracellular $\mathrm{Ca}^{2+}$ concentration; IRS, insulin receptor substrate; $k d A k t$, kinase-dead Akt 1 PKB, protein kinase B; RIP, rat insulin I promoter.

Conflict of interest: The authors have declared that no conflict of interest exists.

Citation for this article: J. Clin. Invest. 114:928-936 (2004)

doi:10.1172/JCI200420016. muscle and fat (17). Ablation of Akt2 in a different background resulted in decreased adipose tissue associated with hyperglycemia and $\beta$ cell failure in a small subset of animals, suggesting that Akt could play a role in $\beta$ cell adaptation to insulin resistance states (18). The generalized defect in conventional knockouts and the existence of 3 different Akt isoforms with similar biochemical characteristics (19) make the alterations in islet $\beta$ cell function in these animal models difficult to interpret. While mice with increased Akt activity exhibit a marked increase in $\beta$ cell mass, this does not define Akt as the physiologic mediator of the effects of the insulin/IGF pathway in $\beta$ cell mass and function $(20,21)$. In order to study the effects of Akt in $\beta$ cell physiology, we have generated transgenic mice expressing a kinase-dead mutant of Akt in $\beta$ cells that serves as a dominant negative inhibitor of Akt activity (22). The results of these experiments clearly demonstrate that Akt is essential for normal $\beta$ cell function and delineate a novel role for Akt in the distal steps of the insulin exocytotic pathway.

\section{Results}

Endogenous Akt1 activity in kinase-dead Akt1 transgenic mice. A kinasedead Akt1 (kdAkt) with mutation at the ATP binding site has been shown previously to act in a dominant negative manner (22-27). The kdAkt sequence was inserted downstream of the rat insulin I promoter (RIP) to generate the transgene (RIP-kdAkt). After transgene microinjection into mouse embryos, 4 founder mice were obtained. Transgene incorporation was demonstrated by Southern blot analysis in each of the lines (data not shown). Progeny from all 4 lines were viable and fertile, and offspring from 2 founders with similar transgene expression and disturbances in glucose tolerance were studied further. The current studies describe the phenotypic characterization of one of these lines. Immunofluorescence staining of pancreatic sections with anti-HA and anti-insulin Ab's showed that the majority of $\beta$ cells (approximately $80 \%$ ) expressed 

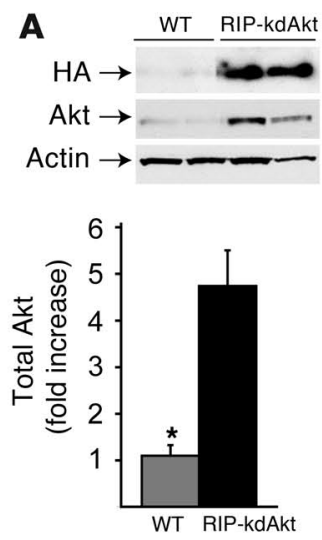

\section{B}

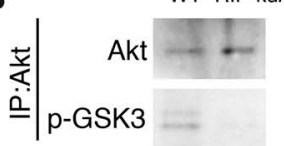

高

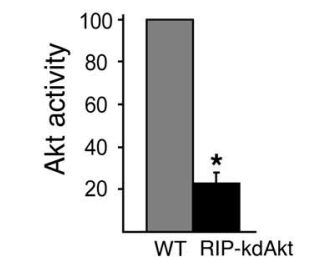

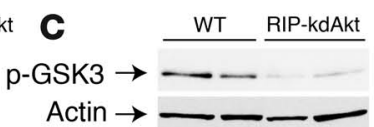

Actin $\rightarrow$

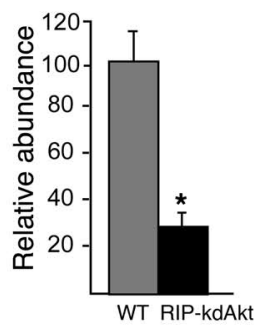

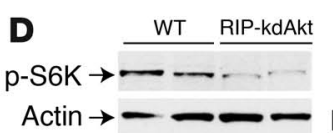
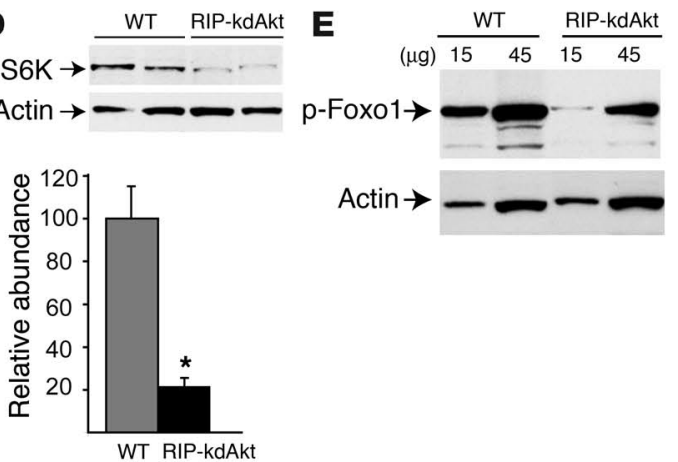

Actin $\rightarrow$

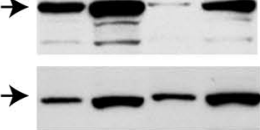

WT RIP-kdAkt

Figure 1

Overexpression of kdAkt inhibits Akt activity and phosphorylation of Akt targets in RIP-kdAkt islets. (A) Total pancreatic islet lysates from RIP-kdAkt and WT mice were immunoblotted with Ab's against HA, Akt, or actin. Semiquantitation of total Akt protein level in islets from RIP-kdAkt and WT mice was adjusted to actin as loading control $(n=6)$. (B) Total in vitro Akt kinase activity from $400 \mu \mathrm{g}$ of islet lysates. Upper blot: Immunoblotting for Akt in the immunoprecipitate. Middle blot and bar graph: In vitro Akt kinase activity assayed by immunoblotting with anti-phospho-GSK3 Ab's (p-GSK3) with semiquantitative analysis $(n=3)$. Lower blot: Immunoblotting for Akt in the post-immunoprecipitation supernatant (Post-IP sup). (C and D) Phosphorylation status in islet lysates and semiquantitative analysis of band intensities for phospho-GSK3 $($ Ser9; $n=5)(\mathbf{C})$ and phospho-S6K (Thr389; $n=6)(\mathbf{D})$ in transgenics and WT mice. (E) Immunoblotting for phospho-Foxo1 (Ser256) and actin in lysates from RIP-kdAkt and WT islets. The data are representative of 3 independent experiments done in duplicate. Data for phospho-GSK3 and phospho-S6K were adjusted to actin and are presented as mean \pm SE. ${ }^{*} P<0.05$.

the transgene. No aberrant expression of the RIP-kdAkt transgene in the hypothalamus was observed by immunostaining of hypothalamic sections with anti-HA Ab (data not shown). Expression of the transgene product in pancreatic islet $\beta$ cells by Western blot analysis using anti-HA Ab's was observed only in RIP-kdAkt mice (Figure 1A). Immunoblotting for Akt showed that the kdAkt protein was expressed in excess $(4.6 \pm 0.8$-fold; $P<0.01, n=6)$ relative to endogenous Akt (Figure 1A).

To assess the specificity and the extent of the inhibition of endogenous Akt by the kdAkt mutant, we determined total in vitro Akt kinase activity and the phosphorylation status of known Akt substrates in islets from these mice. To assess in vitro Akt activity, islet lysates were immunoprecipitated with anti-Akt Ab. The ratio of Akt protein levels in islet lysates from RIP-kdAkt mice and controls after immunoprecipitation was similar to that observed in lysates before immunoprecipitation (data not shown), which suggests that the Akt Ab does not preferentially precipitate the mutant Akt and that the level of this protein is not saturating the immunoprecipitation of the WT Akt (Figure 1B, upper blot). Activity of endogenous Akt in transgenic mouse pancreatic islets was significantly attenuated $(73 \% \pm 5 \% ; P<0.05)$ in RIP-kdAkt mice (Figure 1B, middle blot and bar graph). Akt protein levels in the post-immunoprecipitation supernatant were not detectable, which suggests that the efficiency of immunoprecipitation using this $\mathrm{Ab}$ is adequate to assay Akt activity and that this $\mathrm{Ab}$ is not selectively immunoprecipitating the mutant protein (Figure 1B, lower blot). Similar results were obtained using a higher concentration of $\mathrm{Ab}$ during immunoprecipitation (data not shown).

The reduction in Akt activity obtained by in vitro kinase assays was associated with decreased endogenous GSK3 $\beta$ (Ser9) phosphorylation $(100 \% \pm 17 \%$ vs. $29 \% \pm 6 \%$ ) (Figure 1C). A significant decrease $(70 \% \pm 8 \% ; P<0.01, n=6)$ in phosphorylation of S6K (Thr389) (Figure 1D) and Foxo1 (Ser256) protein (Figure 1E) was observed in lysates from RIP-kdAkt islets. Parentheti- cally, phosphorylation of extracellular signal-regulated kinase (p44/p42), a parallel kinase signaling pathway, was not altered in RIP-kdAkt islets (data not shown). These observations indicated that overexpression of the kdAkt transgene was associated with decreased Akt activity in $\beta$ cells.

RIP-kdAkt mice exhibit impaired glucose tolerance and defective insulin secretion. To determine whether the decrease in Akt activity induced abnormalities in glucose or lipid metabolism, blood samples from 4-month-old RIP-kdAkt and WT littermates were analyzed both in the fasted and the fed states. In the fasting state, blood glucose, plasma insulin concentrations, cholesterol, triglycerides, and FFAs were similar in RIP-kdAkt and WT mice (Table 1). In contrast, RIP-kdAkt mice exhibited hyperglycemia and significantly lower plasma insulin levels in the fed state (Table 1).

Higher fed glucose with lower fasting insulin in the RIP-kdAkt mice suggested that these mice might have impaired glucose tolerance along with a defect in insulin secretion. Glucose tolerance tests were therefore performed in RIP-kdAkt transgenic and WT mice. In the 6- to 8-week-old fasted mice, blood glucose concentrations did not differ between RIP-kdAkt mice and WT littermates (Figure 2A). Blood glucose levels were similar 30 and 60 minutes after

Table 1

Serum parameters in 4-month-old males

\begin{tabular}{lcccc}
\hline & \multicolumn{2}{c}{ Fed } & \multicolumn{2}{c}{ Fasting } \\
& WT & RIP-kdAkt & WT & RIP-kdAkt \\
& $(\boldsymbol{n}=\mathbf{1 9})$ & $(\boldsymbol{n}=\mathbf{1 6})$ & $(\boldsymbol{n}=\mathbf{1 8})$ & $(\boldsymbol{n}=\mathbf{1 0})$ \\
Glucose $(\mathrm{mg} / \mathrm{dl})$ & $188 \pm 5$ & $204 \pm 4 \mathrm{~A}$ & $97 \pm 2$ & $100 \pm 5$ \\
Insulin $(\mathrm{ng} / \mathrm{ml})$ & $1.7 \pm 0.2$ & $1.2 \pm 0.1^{\mathrm{A}}$ & $0.7 \pm 0.01$ & $0.6 \pm 0.01$ \\
Cholesterol $(\mathrm{mg} / \mathrm{dl})$ & ND & ND & $26 \pm 3$ & $22 \pm 3$ \\
Triglycerides (mg/dl) & ND & ND & $79.3 \pm 8$ & $66.5 \pm 6$ \\
FFAs (mmol/l) & ND & ND & $1.8 \pm 0.08$ & $1.6 \pm 0.05$
\end{tabular}

AP $<0.05$. ND, not determined. 

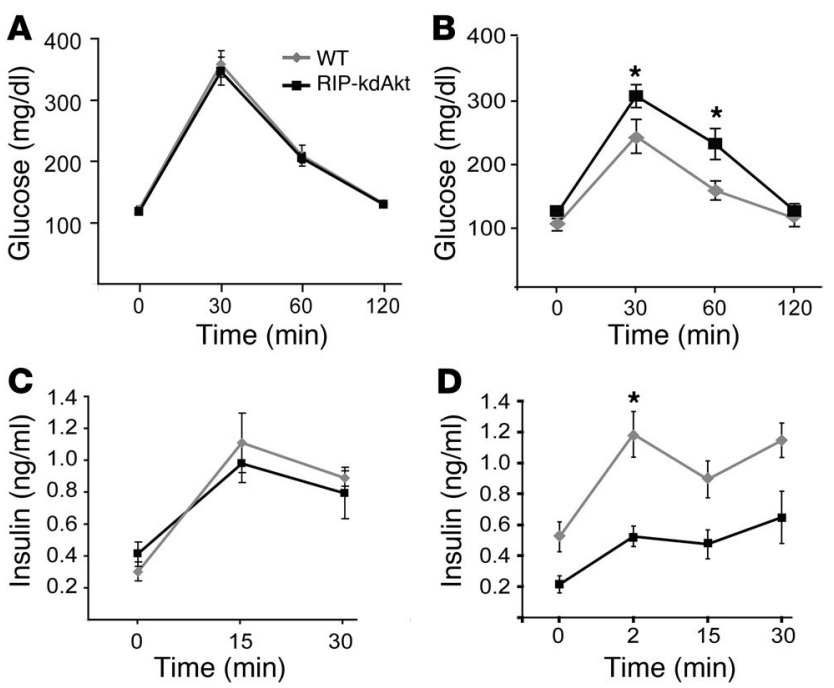

intraperitoneal administration of glucose (Figure 2A) and returned to fasting levels in both groups by 120 minutes. Intraperitoneal glucose tolerance tests performed in 6-month-old mice showed higher glucose levels at 30 and 60 minutes in RIP-kdAkt mice than in controls (Figure 2B; $P<0.05$ ). Males and females from the other transgenic line showed similar results (data not shown). These results suggest that transgenic mice overexpressing a kinase-dead mutant of Akt exhibit abnormal carbohydrate metabolism that becomes more pronounced with aging.

To elucidate the mechanism associated with the alterations in carbohydrate metabolism in RIP-kdAkt mice, glucose-stimulated insulin secretion was evaluated. Similar insulin secretory response to intraperitoneal glucose was observed between RIP-kdAkt and WT littermates at 6-8 weeks of age (Figure 2C). In contrast to the normal fasting insulin levels observed in younger mice (Table 1 and Figure 2C), 6-month-old mice exhibited significant differences in the fasted state (Figure $2 \mathrm{D} ; P<0.05$ ). Additionally, transgenic mice exhibited abnormal insulin release in response to intraperitoneal glucose (Figure 2D).

In vitro assessment of insulin secretion. To assess whether RIP-kdAkt mice have alterations in glucose-stimulated insulin secretion in vitro, islet perifusion experiments were performed (Figure 3A).

\section{Figure 2}

Expression of kdAkt in $\beta$ cells results in impaired glucose tolerance and defective insulin secretion. (A and $\mathbf{B}$ ) Intraperitoneal glucose tolerance tests were performed on 6- to 8-week-old $(\mathbf{A})$ and 6-month-old (B) RIP-kdAkt and WT male mice fasted for $15-18$ hours $(n \geq 5)$. (C and D) Insulin secretion in vivo after intraperitoneal glucose $(3 \mathrm{~g} / \mathrm{kg})$ in 6- to 8-week-old (C) and 6-month-old (D) RIP-kdAkt and WT male mice $(n \geq 5)$. Data are presented as mean \pm SE. ${ }^{*} P<0.05$.

Islets from transgenic mice showed reduced basal (2 mM glucose) insulin secretion in a perifusion system (Figure 3A). Stimulation with $20 \mathrm{mM}$ glucose demonstrated a significant reduction in the early phase and late phase of insulin secretion in islets from RIPkdAkt mice (Figure 3A; $P<0.05$ ). The first and second phases of insulin secretion stimulated by $\mathrm{KCl}$-induced depolarization were also impaired in RIP-kdAkt mice, which suggests that the defect in insulin secretion is distal to $\mathrm{Ca}^{2+}$ entry through voltage-gated $\mathrm{Ca}^{2+}$ channels (Figure 3B). To determine whether the defect in insulin secretion was a generalized defect and not secondary to local concentration of $\mathrm{Ca}^{2+}$ in the proximity of the voltage-gated $\mathrm{Ca}^{2+}$ channels, we used the calcium ionophore ionomycin. Severe impairment in insulin secretion was observed in RIP-kdAkt islets exposed to stepwise increases in ionomycin concentration, which suggests that islets from RIP-kdAkt mice exhibit a major defect in $\mathrm{Ca}^{2+}$-dependent exocytosis (Figure 3C). Glucagon levels measured in the samples collected during ionomycin treatment demonstrated normal responses in RIP-kdAkt islets compared with those in WT mice (area under the curve during ionomycin treatment: $286 \pm 100$ vs. $358 \pm 130 \mathrm{pg} / \mathrm{ml}$, respectively), suggesting that the secretory defect in islets is specific for cells expressing the transgene. These experiments indicated that glucose intolerance in RIP-kdAkt mice results from defective insulin secretion and suggest that Akt plays a major role in insulin exocytosis.

Islet morphology. Abnormalities in glucose tolerance and insulin secretion can result from alterations in islet $\beta$ cell architecture, islet mass, or insulin biosynthesis. Islets from transgenic mice exhibited normal distribution of non- $\beta$ cells at the periphery and insulin-positive cells in the islet core (Figure 4A). Quantitative morphometry showed comparable $\beta$ cell mass (Figure 4B) and islet size distribution (Figure 4C) in RIP-kdAkt mice. Islet $\beta$ cell size in RIP-kdAkt mice was not different from that in the WT mice $\left(92.6 \pm 7\right.$ vs. $\left.99.7 \pm 9 \mu \mathrm{m}^{2}, n=3\right)$. The total pancreatic insulin
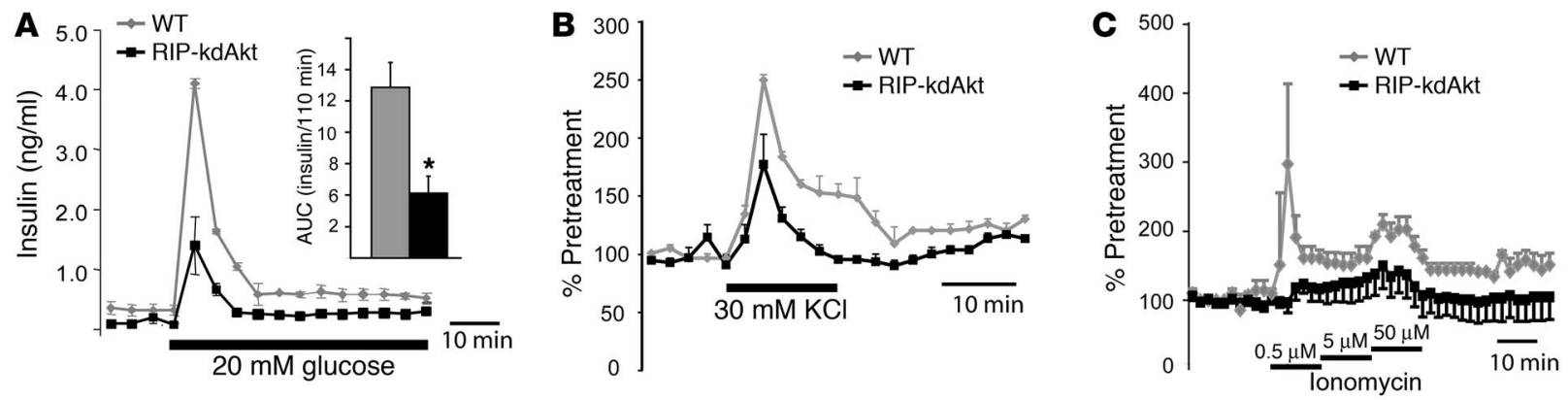

Figure 3

RIP-kdAkt mice exhibit abnormal insulin secretion in vitro. (A) Assessment of insulin secretion by islet perifusion experiments with low (2 mM) and high $(20 \mathrm{mM})$ glucose in islets from RIP-kdAkt and WT mice $(n=3)$. Net insulin-release response, quantified by integration of the area under the curve (AUC) during the treatment period, is shown in the inset. (B) Assessment of insulin secretion by islet perifusion experiments after stimulation with $\mathrm{KCl}(30 \mathrm{mM})$ in islets from RIP-kdAkt and WT mice $(n=3)$. (C) Exposure of RIP-kdAkt and WT islets to step increases in ionomycin concentration in perifusion experiments $(n=3)$. Data are presented as mean $\pm \mathrm{SE}$. ${ }^{*} P<0.05$. 
A
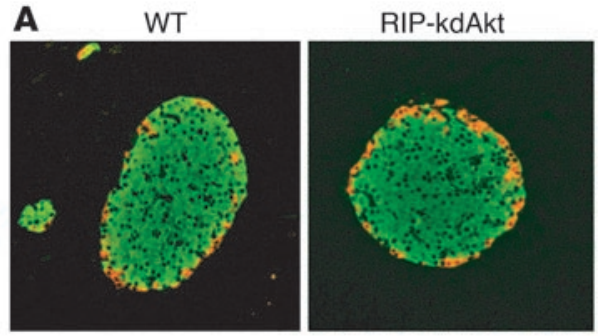

B

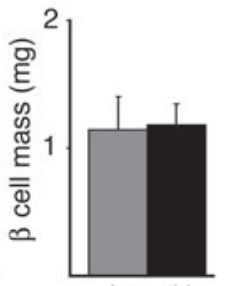

C

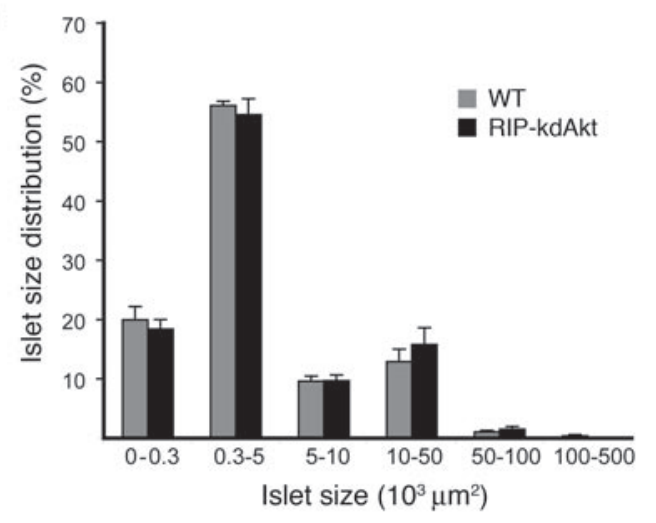

the kinetics of glucose- and $\mathrm{KCl}$-induced insulin secretion (Figure 5, D and $\mathrm{E}$ ) and the percentage of cells responding to these agents were similar (WT, 87\%, vs. RIP-kdAkt, 80\%; $P=$ NS). Specifically, the average response ( $257 \pm 14$ vs. $271 \pm 28 \mathrm{nM}$ ), peak response ( $312 \pm 18$ vs. $317 \pm 28 \mathrm{nM})$, and time to peak $(552 \pm 68$ vs. $549 \pm 108$ seconds) were not significantly different between WT and RIP-kdAkt $\beta$ cells, respectively. RIP-kdAkt $\beta$ cells also exhibited normal oscillatory behavior at $10 \mathrm{mM}$ glucose. The $\left[\mathrm{Ca}^{2+}\right]_{\mathrm{i}}$ responses of islets and disperse $\beta$ cells to $\mathrm{KCl}$-induced depolarization were comparable, indicating that the expression and function of voltage-gated $\mathrm{Ca}^{2+}$ channels was not impaired in RIP-kdAkt $\beta$ cells. The results of these studies suggest that the abnormal insulin secretory phenotype does not lie in proximal steps of glucose sensing, $\beta$ cell metabolism, or $\mathrm{Ca}^{2+}$ influx. Since $\mathrm{Ca}^{2+}$ influx through voltage-gated $\mathrm{Ca}^{2+}$ channels is directly coupled to insulin granule exocytosis, these results point to the requirement of Akt activity in the maintenance of the distal exocytotic pathway.

Insulin secretory proteins are critical for the distal effects of insulin secretion mediated by $\mathrm{Ca}^{2+}$. Protein levels for some of the regulatory proteins of the secretory machinery were assessed to determine whether the defects in insulin secretion observed in RIP-kdAkt mice resulted from abnormal expression of these proteins. Protein expression for the regulatory proteins Rab3a, synaptotagmin, and Munc18 was not altered in islets from RIPkdAkt transgenic mice (Figure 5F). Expression of the soluble NSF attachment protein receptor syntaxin 6 was no different in RIPkdAkt islets than in WT (Figure 5F).

Adaptive responses to a bigh-fat diet in RIP-kdAkt mice. To determine whether Akt is important for the $\beta$ cell adaptive responses to insulin resistance, 9-month-old WT and RIP-kdAkt mice were fed a high-fat diet for 15 weeks. RIP-kdAkt mice gained weight to the same extent as WT littermates (Table 3). In contrast to the normal fasting glucose and insulin levels observed at 4 months of age (Table 1), 9-month-old RIP-kdAkt mice exhibited significant hyperglycemia and hypoinsulinemia in the fasting state before fat feeding (Table 3). RIP-kdAkt mice exhibited significant hyperglycemia and hypoinsulinemia compared with WT mice after 4 and 15 weeks of fat feeding, and the glucose/insulin ratio decreased significantly, indicating the presence of insulin resistance in both groups. Table 3 also shows that the magnitude of the change following fat feeding was similar. Cholesterol levels

\section{Table 2}

Insulin content in RIP-kdAkt and control mice in glucose from 2 to $26 \mathrm{mM}$ over 50 minutes and a single-step increase in $\mathrm{KCl}$ to $20 \mathrm{mM}$ (Figure 5, B and C). Comparable $\left[\mathrm{Ca}^{2+}\right]_{\mathrm{i}}$ responses to single-step increase in glucose from 2 to $20 \mathrm{mM}$ were also observed (data not shown). These findings suggest that the glucose-sensing threshold and maximal responses are unaffected by Akt inhibition. Experiments performed in single $\beta$ cells from RIP-kdAkt mice and WT mice also demonstrated that

\begin{tabular}{lrc}
\hline & WT & RIP-kdAkt \\
Pancreatic insulin content $(\mathrm{mg})(n=10)$ & $13.7 \pm 2.6$ & $15.3 \pm 1.9$ \\
Insulin content from isolated islets & $6.4 \pm 0.1$ & $7.6 \pm 1$ \\
$\quad(\mathrm{ng} / \mu \mathrm{g})(n=3)$ & & \\
\hline
\end{tabular}



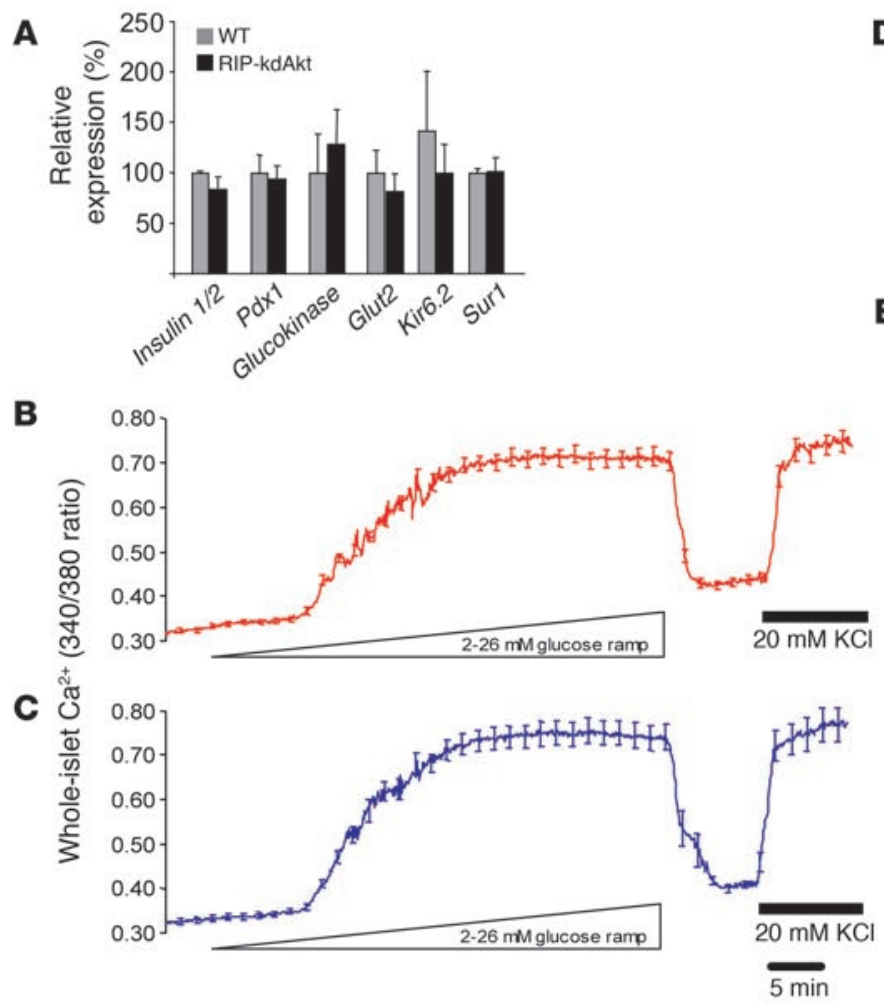

D

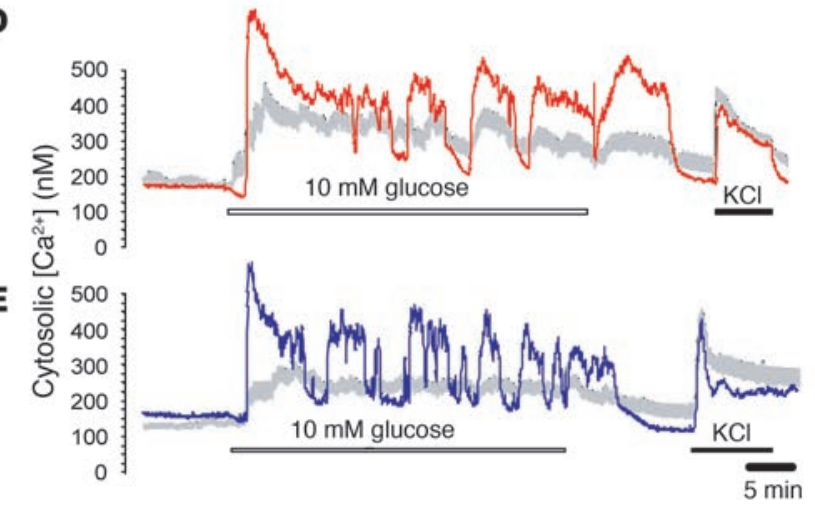

$\mathbf{F}$

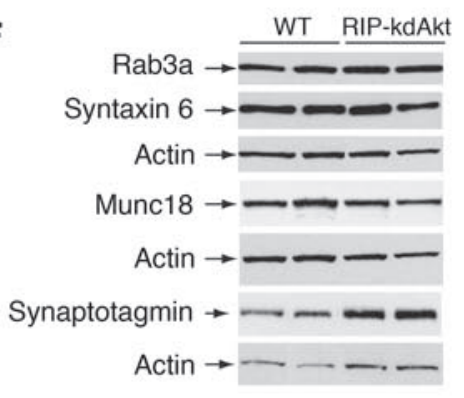

Figure 5

RIP-kdAkt mouse islets exhibit similar gene expression, normal intracellular calcium responses to glucose and $\mathrm{KCl}$, and comparable secretory protein levels. (A) Gene expression by real-time RT-PCR in islets from RIP-kdAkt and WT mice $(n=3)$. (B and C) Measurements of whole-islet $\mathrm{Ca}^{2+}$ induced by a progressive increase in glucose concentration from 2 to $26 \mathrm{mM}$ over 50 minutes followed by an increase in $\mathrm{KCl}$ to $20 \mathrm{mM}$ in isolated islets from RIP-kdAkt (B) and WT (C) mice. (D and E) Measurements of intracytosolic $\mathrm{Ca}^{2+}$ induced by $10 \mathrm{mM}$ glucose and $20 \mathrm{mM} \mathrm{KCl}$ in single $\beta$ cells from RIP-kdAkt (D) and WT (E) islets. Representative tracing is depicted in red (RIP-kdAkt) and blue (WT), and average cytosolic $\mathrm{Ca}^{2+}$ responses are observed in gray $(n=10-15)$. (F) Secretory protein expression by Western blotting in RIP-kdAkt and WT islets. The experiment is representative of 3 independent experiments done in duplicate.

were increased to the same extent in both groups after fat feeding. Triglyceride and FFA levels did not differ between the 2 groups after fat feeding (data not shown). In contrast to the results shown in Table 3, responses to intraperitoneal glucose tolerance tests after 12 weeks of fat feeding demonstrated that RIP-kdAkt mice developed severe hyperglycemia that persisted after $120 \mathrm{~min}$ utes of glucose administration (Figure 6A). Similar compensatory responses of $\beta$ cell mass to insulin resistance were observed in RIP-kdAkt mice by quantitative morphometry of islet area (Figure 6B). Distribution of the frequency of islets by size demonstrated a similar frequency of islets except that islet sizes less than $30 \times 10^{3}$ $\mu \mathrm{m}^{2}$ (islets with 1-3 cells) were less abundant in RIP-kdAkt mice $(11.6 \% \pm 1.3 \%$ vs. $17 \% \pm 1.4 \%)$ (Figure $6 \mathrm{C}$ ). The results of these experiments suggest that overexpression of a kinase-dead Akt impairs the adaptation of $\beta$ cells to obesity-induced insulin resistance mainly via an insulin secretory defect and not by alterations in the capacity to increase $\beta$ cell mass.

In vitro apoptosis studies in islets from RIP-kdAkt mice. The normal $\beta$ cell mass observed in RIP-kdAkt mice in the basal state or under conditions of insulin resistance suggested that apoptosis was not a major contributor in this phenotype. As shown in Figure 7A, the frequency of activated caspase-3-positive cells in pancreatic sections from 6-month-old RIP-kdAkt mice was not different from that of WT mice. However, islets from RIP-kdAkt mice could have increased susceptibility to apoptosis when exposed to known apoptotic agents. To assess the susceptibility to apoptosis of RIPkdAkt islets in vitro, we evaluated the effect of known apoptotic stimuli in vitro using PCR-enhanced DNA laddering. Susceptibility to apoptosis was not different in islets from RIP-kdAkt and WT mice during basal culture conditions (10 $\mathrm{mM}$ glucose) for 48 hours (Figure 7B). Culture of islets from RIP-kdAkt mice with $1 \mu \mathrm{M}$ thapsigargin, a known inducer of the endoplasmic reticulum stress pathway, resulted in a degree of apoptosis similar to that in islets from WT mice (Figure 7B) (28-30). Comparable apoptosis between RIP-kdAkt mice and WT islets was observed after culture in serum-free medium for 7 days (Figure 7C). Susceptibility to apoptosis was similar between the 2 groups when islet apoptosis was induced by $250 \mu \mathrm{M}$ palmitate (data not shown). The results of these experiments suggest that overexpression of the kinase-dead mutant of Akt does not play a dominant role in controlling islet survival under these conditions.

\section{Discussion}

The results of the current studies serve to elucidate the mechanisms involved in the regulation of $\beta$ cell function by Akt. These findings indicate that the serine-threonine kinase Akt is necessary for normal pancreatic $\beta$ cell function and describe a novel regulatory role for Akt signaling in insulin secretion at the level of the 
Table 3

Weight and fasting metabolic parameters before and after fat feeding

\begin{tabular}{lccrcc} 
& $\begin{array}{c}\text { Weight } \\
(\mathbf{g})\end{array}$ & $\begin{array}{c}\text { Glucose } \\
(\mathbf{m g} / \mathbf{d l})\end{array}$ & $\begin{array}{c}\text { Insulin } \\
(\mathbf{n g} / \mathbf{m l})\end{array}$ & $\begin{array}{c}\text { Glucose/insulin ratio } \\
\text { (ratio mg/ng })\end{array}$ & $\begin{array}{c}\text { Cholesterol } \\
(\mathbf{m g} / \mathbf{d l})\end{array}$ \\
WT & & & & & \\
Before & $43 \pm 1.5$ & $133 \pm 6.7$ & $5.6 \pm 0.7$ & $26 \pm 3$ & $42.1 \pm 7.7$ \\
4 weeks & $50 \pm 1.4$ & $165 \pm 7$ & $10.1 \pm 1.3$ & $18.4 \pm 2$ & $123.9 \pm 11$ \\
15 weeks & $51 \pm 0.8$ & $159 \pm 10$ & $9.3 \pm 0.9$ & $16.3 \pm 3$ & $207.4 \pm 26$ \\
RIP-kdAkt & & & & & \\
Before & $39 \pm 1.8$ & $163 \pm 8^{\mathrm{A}}$ & $3.1 \pm 0.5^{\mathrm{A}}$ & $47.5 \pm 7.3^{\mathrm{A}}$ & $50.3 \pm 6.7$ \\
4 weeks & $49 \pm 1.6$ & $193 \pm 8^{\mathrm{A}}$ & $8.9 \pm 1.4^{\mathrm{A}}$ & $25.5 \pm 3.5^{\mathrm{A}}$ & $124 \pm 9$ \\
15 weeks & $49 \pm 1.8$ & $198 \pm 14^{\mathrm{A}}$ & $7.7 \pm 1.8^{\mathrm{A}}$ & $27.8 \pm 3.4^{\mathrm{A}}$ & $220 \pm 31$ \\
\hline
\end{tabular}

Results are expressed as mean \pm SEM from at least 6 male mice per group. ${ }^{A} P<0.05$ by ANOVA between RIP-kdAkt and WT. For all parameters, the values after fat feeding were significantly different from the values before fat feeding $(P<0.01)$.

exocytotic pathway distal to $\mathrm{Ca}^{2+}$ influx. These observations also suggest that the $80 \%$ reduction in Akt activity obtained in this experimental model is not associated with decreased $\beta$ cell mass, under normal conditions or under conditions of insulin resistance induced by fat feeding. Deterioration of the glucose intolerance associated with insulin resistance induced by aging and fat feeding suggests that Akt activity might be an important component of adaptive responses of $\beta$ cells to insulin resistance, and to resistance to experimental diabetes.

The in vivo experiments indicated that glucose tolerance in RIPkdAkt animals was impaired at 30 and 60 minutes (Figure 2B). Insulin secretion in response to an intraperitoneal glucose load (Figure 2D) suggested that the basal insulin secretion was reduced, but the increment did not differ in RIP-kdAkt mice compared with WT. However, the similar insulin secretory response to a greater glycemic stimulus following glucose challenge in transgenic animals indicates that the insulin secretion in response to glucose was impaired. The defective insulin response induced by glucose and $\mathrm{KCl}$ in perifusion experiments further confirmed the in vivo findings (Figure 3, A and B). Alterations in insulin secretion in the presence of normal $\left[\mathrm{Ca}^{2+}\right]_{\mathrm{i}}$ responses induced by glucose and $\mathrm{KCl}$ (Figure 5, B and C) in islets and dispersed $\beta$ cells from RIPkdAkt mice (Figure 5, D and E) indicated that the mechanism of the secretory defect was distal to $\mathrm{Ca}^{2+}$ influx and not associated with alterations in glucose sensing. Finally, the results of the experiments with ionomycin (Figure 3C) implied that the defect in $\mathrm{Ca}^{2+}$-dependent insulin exocytosis was diffuse and that it could not be explained by alterations in the function of the voltage-gated $\mathrm{Ca}^{2+}$ channels or in the interactions of these channels with proteins of the exocytotic machinery. Further, these results suggested that long-term inhibition of Akt signaling in $\beta$ cells does not alter proximal steps of glucose signaling and function of voltage-gated $\mathrm{Ca}^{2+}$ channels and that the mechanisms involved in regulation

\section{Figure 6}

Deterioration of the glucose intolerance associated with insulin resistance induced by fat feeding in RIP-kdAkt mice. (A) Intraperitoneal glucose tolerance test in 12-week-old RIP-kdAkt and WT mice after 12 hours of fasting $(n=7)$. (B) Islet area after 14 weeks of fat feeding in RIP-kdAkt and WT mice $(n=6)$. (C) Islet size distribution obtained from mice used for $\beta$ cell mass measurements. Data are presented as mean \pm SE. ${ }^{*} P \leq 0.05$. of insulin secretion in RIP-kdAkt mice lie at the level of insulin exocytosis. It is still possible that the secretory defect is secondary to alterations in PDK activity, since overexpression of kdAkt could theoretically inhibit downstream targets of PDK. However, no obvious alteration in PDK activity was observed when the kdAkt protein was overexpressed in cardiac myocytes (31). The results of these observations highlight the importance of the insulin signaling pathway in the long-term regulation of insulin secretion and suggest that Akt is a major component mediating these events.

The distal abnormalities in insulin secretion found by overexpression of the kdAkt protein could result from alterations in the levels or function of exocytotic machinery proteins. Moreover, a similar defective exocytotic mechanism has been described in mice deficient in the GTP-binding protein Rab3A (32). Levels of Rab3A and other regulatory proteins important for insulin secretion, such as synaptotagmin and Munc18, were not altered in islets from RIP-kdAkt mice (Figure 5F). It is still possible that Akt regulates the function of some of these proteins by phosphorylation or by modulating interaction with other proteins involved in vesicle trafficking. Trafficking of insulin granules to the cell surface requires components of the cytoskeletal framework of microtubules and actin filaments (33). Recent evidence indicated that the PI3K/Akt pathway can bind and regulate $(34,35)$ actin filament remodeling, which suggests that this may be a mechanism by which Akt regulates insulin exocytosis. Further experiments should be carried out to test this hypothesis.

The observation that overexpression of a constitutively active form of Akt in islet $\beta$ cells results in striking increases in $\beta$ cell mass suggests that Akt is important for growth responses, but it does not provide insight into the role of endogenous Akt in $\beta$ cell physiology $(20,21)$. The current studies were designed to

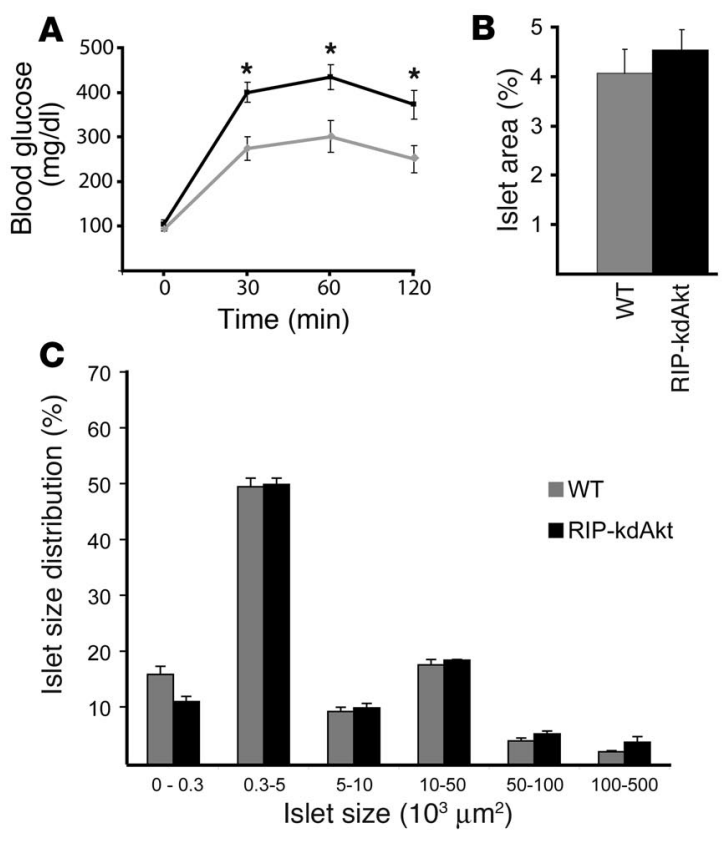



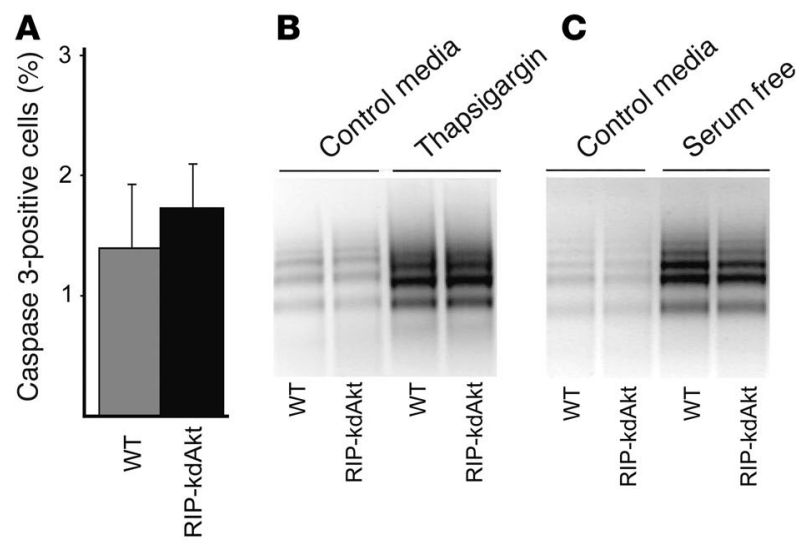

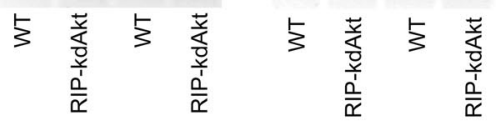

\section{Figure 7}

Assessment of islet apoptosis in RIP-kdAkt and WT mice. (A) Frequency of activated caspase-positive cells in islets from RIP-kdAkt and WT mice $(n=3)$. (B) DNA laddering in RIP-kdAkt and WT islets after 48 hours of incubation in RPMI containing $10 \mathrm{mM}$ glucose, $10 \%$ serum (control medium), and $1 \mu \mathrm{M}$ thapsigargin. (C) Apoptosis assessment by DNA laddering in RIP-kdAkt and WT islets cultured for 7 days in control medium and in serum-free RPMI with $10 \mathrm{mM}$ glucose. Data are representative of at least 3 experiments in duplicate.

evaluate the role of endogenous Akt signaling in $\beta$ cells in the absence of alterations in peripheral tissues. These experiments showed that overexpression of kdAkt mutant in $80 \%$ of the $\beta$ cells was not associated with alterations in $\beta$ cell mass or cell size in normal conditions or conditions of fat feeding. It is still possible that $20 \%$ residual Akt function in RIP-kdAkt mice is sufficient to preserve $\beta$ cell mass even under conditions of insulin resistance such as those following high-fat feeding. Evidence that endogenous Akt is important for the regulation of $\beta$ cell mass comes from experiments with Foxo1, a transcription factor that is negatively regulated by Akt. In these experiments, the diabetes and $\beta$ cell failure of IRS2-deficient mice were reversed by Foxo1 haploinsufficiency (36). Further, while Akt2-deficient mice did not appear to have alterations in $\beta$ cell mass, a more recent study in a different genetic background resulted in severe diabetes and $\beta$ cell failure in a small subgroup of animals (18). Thus, the direct role of Akt in the regulation of $\beta$ cell mass under conditions of insulin resistance is not completely defined. Experiments in mice lacking the 3 isoforms of Akt in $\beta$ cells might better determine the role of Akt in the regulation of $\beta$ cell mass and function.

In conclusion, the results of these experiments demonstrate an Akt-dependent mechanism in the distal events of insulin exocytosis and suggest that alteration of Akt signaling in $\beta$ cells can result in an insulin secretory dysfunction similar to those observed in type 2 diabetes. These observations also suggest that pharmacologic modulation of Akt and proteins of the exocytotic machinery could potentially provide novel therapeutic targets for the improvement of $\beta$ cell dysfunction in type 2 diabetes.

\section{Methods}

Generation of transgenic mice. The kinase-dead Akt1 (Akt K179M) (22) was inserted in a RIP-I/ $\beta$-globin expression vector and microinjected into fertilized eggs of C57BL/6 $\times$ CBA mice according to standard technique (37). Four transgenic founders were obtained and backcrossed to C57BL/6J mice (The Jackson Laboratory), and the N2 and N3 generations from 2 lines with similar phenotype were analyzed. WT littermates were used as controls for all the experiments. All procedures were performed in accordance with Washington University's Animal Studies Committee.

Western blotting and in vitro Akt activity. For Western blot analysis, blots of isolated pancreatic islet lysates were probed with Ab's against HA epitope (Covance Inc.), actin (Sigma-Aldrich), phospho-GSK3 $\beta$ (Ser9), phospho-p70 S6K (Thr389), phospho-Foxo1 (Ser256), Akt (Cell Signaling Technology Inc.), Rab3a (Abcam Ltd.), syntaxin 6, Munc18, and synaptotagmin (BD Biosciences). For in vitro Akt kinase assay, $400 \mu \mathrm{g}$ of islet lysates from 8 RIP-kdAkt and 8 WT mice was studied using the Akt Kinase Assay Kit (Cell Signaling Technology Inc.) according to the manufacturer's protocol. The $\mathrm{Ab}$ used for this assay was generated using a synthetic phosphopeptide corresponding to residues around 473 of human Akt and recognizes Akt $1, \mathrm{Akt} 2$, and Akt 3 isoforms. Akt activity was measured by phosphorylation of GSK3 $\alpha / \beta$ through Western blotting using a phospho-GSK3 $\alpha / \beta$ (Ser21/9) Ab. Immunoblotting for immunoprecipitated Akt was performed in the same gel used to assess Akt activity. Efficiency of immunoprecipitation for Akt was determined by comparing Akt protein levels from islet lysates before immunoprecipitation with those obtained in the supernatant after immunoprecipitation with the recommended amount of $\mathrm{Akt} \mathrm{Ab}$ and with 8-fold excess $\mathrm{Ab}$. Scanning densitometry of protein bands was determined by pixel intensity using NIH ImageJ software (v1.31; freely available at http://rsb.info.nih.gov/ij/docs/install) and normalized against that of actin.

Immunohistochemistry and immunofluorescence. Pancreata were fixed overnight in Bouin's solution, and embedded in paraffin using standard techniques. Immunostaining for insulin was done with guinea pig anti-human insulin $\mathrm{Ab}$ (BioGenex) and the HistoMouse-SP kit (Zymed Laboratories Inc.) using 3-amino-9-ethyl carbazole (AEC) as chromogen according to the manufacturers' instructions. For immunofluorescence, primary Ab's were used as described previously (21).

Quantitative real-time RT-PCR. Total RNA was isolated from purified islets using the RNeasy isolation kit (QIAGEN Inc.) and reverse-transcribed using SuperScript II (Invitrogen Corp.). Quantitative real-time RT-PCR was performed using SYBR Green dye (ABI) in an ABI 7000 detection system (Applied Biosystems). Relative abundance for each transcript was calculated by a standard curve of cycle thresholds for serial dilutions of a cDNA sample and normalized to $18 \mathrm{~S}$ ribosomal RNA.

Islet morphometry analysis and total pancreatic insulin content. Pancreata were obtained from 4-month-old WT and transgenic mice for immunohistochemistry. The $\beta$ cell mass was calculated by point-counting morphometry from 5 insulin-stained sections $(5 \mu \mathrm{m})$ separated by 200 $\mu \mathrm{m}$ using the BIOQUANT Classic98 software package (BIOQUANT Image Analysis Corp.) as described previously (21). Islet $\beta$ cell size was calculated as previously described (38) by counting of at least $1,000 \beta$ cells per animal. Measurement of pancreatic and islet insulin content was performed by acid ethanol extraction as described previously (38).

Metabolic studies. Fasting blood samples were obtained from the tail vein after overnight fasting. Fed glucose measurements were performed between 9 and $10 \mathrm{am}$. For the fat-feeding experiments, blood samples were obtained from mice fasted for 6 hours. Glucose was measured in whole blood using the Accu-Chek II glucometer (Roche Diagnostics). Plasma insulin levels were determined in 5- $\mu \mathrm{l}$ aliquots using a rat insulin ELISA kit (Crystal Chem Inc.). Glucose tolerance tests were performed in animals fasted for 12 hours, by intraperitoneal injection of glucose $(2 \mathrm{mg} / \mathrm{g})$ as described previously (21). Glucose tolerance tests for the fat-feeding experiment were performed on mice fasted for 6 hours. For insulin secretion, mice were injected with glucose $(3 \mathrm{mg} / \mathrm{g})$ as described for the glucose tolerance test (21). Fat-feeding experiments were performed in 9-month-old mice using chow containing total fat content of 6\% (TD 88137; Harlan Teklad). 
Islet isolation and insulin secretion from isolated perifused pancreatic islets. Islet isolation was accomplished by collagenase digestion (21). Islet perifusion experiments were carried out as described previously (39). Briefly, groups of 80 were suspended in Bio-Gel P2 beads and perifused at $1 \mathrm{ml} / \mathrm{min}$ using a temperature-controlled multichamber perifusion system (Cellex Biosciences Inc.). In some cases, insulin measurement values from perifusion conditions are normalized to the mean of the first 5 measurements (percent pretreatment). Net hormone-release responses of perifused cell columns to treatments were quantified by integration of the base-line subtracted area under the curve during the treatment period. Each time point was subtracted from the prepulse mean, defined as the average of the 3 time points before the treatment period.

Intracellular free $\mathrm{Ca}^{2+}$ measurements from isolated islets and single cells. Changes in intracellular $\mathrm{Ca}^{2+}$ within individual islets were measured as described previously (40). Briefly, groups of islets loaded with fura-2-AM for $30 \mathrm{~min}$ utes were imaged using a Nikon inverted microscope (Nikon Inc.) and MetaFluor software (Universal Imaging Corp.). Computer-controlled Gilson minipumps generated ramp protocols as described previously $(39,41)$. For single-cell studies, islets were gently dispersed and plated on coverslips as described previously (42). $\mathrm{Ca}^{2+}$ signals were measured in cells loaded with fura-4F-AM (Invitrogen Corp.); the signals were continuously perifused and excited at $340 \mathrm{nM}$ and $380 \mathrm{nM}$ with a charged coupled device (CCD) camera (TILL Photonics LLC) (42), and then quantified as described previously (43). $\mathrm{Ca}^{2+}$ levels during the 5 minutes prior to treatment were averaged to give a pretreatment mean for each cell. The maximum $\mathrm{Ca}^{2+}$ determination during the treatment period was subtracted from the pretreatment average to give the maximal amplitude of the $\mathrm{Ca}^{2+}$ signal. The time of the maximal amplitude was subtracted from the time of onset of the treatment to give the time to maximal amplitude. The maximal amplitude and time to maximal amplitude were divided to give the effective rate of rise of the $\mathrm{Ca}^{2+}$ signal.

Assessment of apoptosis in vitro. Activated caspase- 3 staining was performed using anti-caspase-3 Ab (BioCarta) as described previously (44). Percentage of activated caspase-3-positive cells was measured in at least 500 islet nuclei from each animal. Islet apoptosis was assayed in vitro using PCR-enhanced DNA ladders, as described previously (45). Briefly, groups of 10 islets were cultured for 48 hours in RPMI 1640 contain- ing $10 \mathrm{mM}$ glucose with or without $10 \%$ FCS as indicated. Apoptosis was induced with $1 \mu \mathrm{M}$ thapsigargin or $250 \mu \mathrm{M}$ palmitate, a known inducer of apoptosis in $\beta$ cells $(28,29)$. To examine the effect of serum withdrawal, islets were cultured for 7 days. Genomic DNA was isolated with the DNeasy kit (QIAGEN Inc.) according to the manufacturer's instructions for cultured cells, except that islets were maintained in lysis buffer for 15 minutes at $55^{\circ} \mathrm{C}$. Genomic DNA was quantified using UV spectrophotometry. Next, DNA was ligated to adapters as described and subjected to short cycles of PCR that selectively amplify adapter-ligated DNA fragments over longer DNA. PCR products were run on $1.2 \%$ agarose/ethidium bromide gels in $0.5 \times$ Tris-borate-EDTA.

Statistical analysis. All values are expressed as mean \pm SEM. Experiments involving multiple sampling times, i.e., the fat-feeding experiments, were analyzed with one-way ANOVA using Analyse-it software (Analyse-it Software Ltd.). For all other comparisons, a nonpaired Student's $t$ test was used. Differences were considered statistically significant at $P<0.05$.

\section{Acknowledgments}

The authors would like to acknowledge the support of the Washington University Diabetes Research and Training Center for insulin and lipid measurements. The authors would like to thank Cris M. Welling and Stacey S. Donelan for technical assistance and Gary Skolnick for preparation of the manuscript. This work was supported in part by an NIH grant (R37 DK16746, to M.A. Permutt), by a Junior Faculty Award from the American Diabetes Association (to E. Bernal-Mizrachi), by a Mentor-Based Fellowship Award from the American Diabetes Association (to S. Fatrai), and by the Juvenile Diabetes Research Foundation (to J.D. Johnson).

Received for publication September 11, 2003, and accepted in revised form August 3, 2004.

Address correspondence to: Ernesto Bernal-Mizrachi, Washington University School of Medicine, 660 S. Euclid Avenue, Campus Box 8127, St. Louis, Missouri 63110, USA. Phone: (314) 362-7693; Fax: (314) 747-2692; E-mail: ebernal@im.wustl.edu.
1. White, M.F. 2002. IRS proteins and the common path to diabetes. Am. J. Physiol. Endocrinol. Metab. 283:E413-E422.

2. Kahn, S.E. 2001. Clinical review 135. The importance of beta-cell failure in the development and progression of type 2 diabetes [review]. J. Clin. Endocrinol. Metab. 86:4047-4058.

3. Bonner-Weir, S. 2000. Life and death of the pancreatic beta cells [review]. Trends Endocrinol. Metab. 11:375-378.

4. Kulkarni, R.N., et al. 1999. Tissue-specific knockout of the insulin receptor in pancreatic beta cells creates an insulin secretory defect similar to that in type 2 diabetes. Cell. 96:329-339.

5. Kido, Y., et al. 2000. Tissue-specific insulin resistance in mice with mutations in the insulin receptor, IRS-1, and IRS-2. J. Clin. Invest. 105:199-205.

6. Withers, D.J., et al. 1998. Disruption of IRS-2 causes type 2 diabetes in mice. Nature. 391:900-903.

7. Xuan, S., et al. 2002. Defective insulin secretion in pancreatic $\beta$ cells lacking type 1 IGF receptor. J. Clin. Invest. 110:1011-1019. doi:10.1172/ JCI200215276.

8. Kulkarni, R.N., et al. 2002. $\beta$-Cell-specific deletion of the Igf1 receptor leads to hyperinsulinemia and glucose intolerance but does not alter $\beta$-cell mass. Nat. Genet. 31:111-115.

9. Buteau, J., et al. 2001. Protein kinase C activation mediates glucagon-like peptide-1-induced pancre- atic $\beta$-cell proliferation. Diabetes. 50:2237-2243.

10. Hugl, S.R., White, M.F., and Rhodes, C.J. 1998 Insulin-like growth factor I (IGF-I)-stimulated pancreatic beta-cell growth is glucose-dependent. Synergistic activation of insulin receptor substrate-mediated signal transduction pathways by glucose and IGF-I in INS-1 cells. J. Biol. Chem. 273:17771-17779.

11. Cousin, S.P., et al. 1999. Stimulation of pancreatic beta-cell proliferation by growth hormone is glucose-dependent: signal transduction via Janus kinase 2 (JAK2)/signal transducer and activator of transcription 5 (STAT5) with no crosstalk to insulin receptor substrate-mediated mitogenic signalling. Biochem. J. 344:649-658.

12. Cousin, S.P., et al. 2001. Free fatty acid-induced inhibition of glucose and insulin-like growth factor I-induced deoxyribonucleic acid synthesis in the pancreatic beta-cell line INS-1. Endocrinology. 142:229-240.

13. Kandel, E.S., and Hay, N. 1999. The regulation and activities of the multifunctional serine/threonine kinase Akt/PKB. Exp. Cell Res. 253:210-229.

14. Toker, A. 2000. Protein kinases as mediators of phosphoinositide 3-kinase signaling. Mol. Pharmacol. 57:652-658.

15. Cho, H., Thorvaldsen, J.L., Chu, Q., Feng, F., and Birnbaum, M.J. 2001. Akt1/PKBalpha is required for normal growth but dispensable for mainte- nance of glucose homeostasis in mice. J. Biol. Chem. 276:38349-38352.

16. Chen, W.S., et al. 2001. Growth retardation and increased apoptosis in mice with homozygous disruption of the Akt1 gene. Genes Dev. 15:2203-2208.

17. Cho, H., et al. 2001. Insulin resistance and a diabetes mellitus-like syndrome in mice lacking the protein kinase Akt2 (PKB beta). Science. 292:1728-1731.

18. Garofalo, R.S., et al. 2003. Severe diabetes, agedependent loss of adipose tissue, and mild growth deficiency in mice lacking Akt2/PKB $\beta$. J. Clin. Invest. 112:197-208.

19. Coffer, P.J., Jin, J., and Woodgett, J.R. 1998. Protein kinase B (c-Akt): a multifunctional mediator of phosphatidylinositol 3-kinase activation. Biochem. J. 335:1-13.

20. Tuttle, R.L., et al. 2001. Regulation of pancreatic beta-cell growth and survival by the serine/threonine protein kinase Akt1/PKBalpha. Nat. Med. 7:1133-1137.

21. Bernal-Mizrachi, E., Wen, W., Stahlhut, S., Welling, C., and Permutt, M.A. 2001. Islet $\beta$ cell expression of constitutively active Akt1/PKB induces striking hypertrophy, hyperplasia, and hyperinsulinemia. J. Clin. Invest. 108:1631-1638. doi:10.1172/JCI200113785.

22. Kohn, A.D., Takeuchi, F., and Roth, R.A. 1996. Akt, a pleckstrin homology domain containing kinase, is activated primarily by phosphorylation. J. Biol. 
Chem. 271:21920-21926.

23. Kennedy, S.G., et al. 1997. The PI 3-kinase/Akt signaling pathway delivers an anti-apoptotic signal. Genes Dev. 11:701-713.

24. Dudek, H., et al. 1997. Regulation of neuronal survival by the serine-threonine protein kinase Akt. Science. 275:661-665.

25. Skorski, T., et al. 1997. Transformation of hematopoietic cells by $\mathrm{BCR} / \mathrm{ABL}$ requires activation of a PI-3k/Akt-dependent pathway. EMBOJ. 16:6151-6161

26. Cheng, H.L., Steinway, M., Delaney, C.L., Franke, T.F., and Feldman, E.L. 2000. IGF-I promotes Schwann cell motility and survival via activation of Akt. Mol. Cell. Endocrinol. 170:211-215.

27. Franke, T.F., et al. 1995. The protein kinase encoded by the Akt proto-oncogene is a target of the PDGF-activated phosphatidylinositol 3-kinase. Cell. 81:727-736.

28. Zhou, Y.P., et al. 2000. Overexpression of Bcl-x(L) in beta-cells prevents cell death but impairs mitochondrial signal for insulin secretion. Am. J. Physiol. Endocrinol. Metab. 278:E340-E351.

29. Zhou, Y.-P., et al. 1998. Apoptosis in insulin-secreting cells. Evidence for the role of intracellular $\mathrm{Ca}^{2}$ stores and arachidonic acid metabolism. J. Clin. Invest. 101:1623-1632.

30. Song, L., De Sarno, P., and Jope, R.S. 2002. Central role of glycogen synthase kinase- $3 \beta$ in endoplasmic reticulum stress-induced caspase- 3 activation. J. Biol. Chem. 277:44701-44708.

31. Shioi, T., et al. 2002. Akt/protein kinase B promotes organ growth in transgenic mice. Mol. Cell. Biol. 22:2799-2809.

32. Yaekura, K., et al. 2003. Insulin secretory deficiency and glucose intolerance in Rab3A null mice. J. Biol. Chem. 278:9715-9721.

33. Lang, J. 1999. Molecular mechanisms and regulation of insulin exocytosis as a paradigm of endocrine secretion. Eur. J. Biochem. 259:3-17.

34. Qian, Y., et al. 2004. PI3K induced actin filament remodeling through Akt and p70S6K1: implication of essential role in cell migration. Am. J. Physiol. Cell Physiol. 286:C153-C163.

35. Cenni, V., et al. 2003. Targeting of the Akt/PKB kinase to the actin skeleton. Cell. Mol. Life Sci. 60:2710-2720.

36. Kitamura, T., et al. 2002. The forkhead transcription factor Foxo1 links insulin signaling to $\mathrm{Pdx} 1$ regulation of pancreatic $\beta$ cell growth. J. Clin. Invest. 110:1839-1847. doi:10.1172/JCI200216857.

37. Hogan, B., Costantini, F., and Lacy, E. 1986. Manipulating the mouse embryo: a laboratory manual. Cold Spring Harbor Laboratory Press. Cold Spring Harbor, New York, USA. 157-173.

38. Montana, E., Bonner-Weir, S., and Weir, G.C. 1993.
Beta cell mass and growth after syngeneic islet cell transplantation in normal and streptozocin diabetic C57BL/6 mice. J. Clin. Invest. 91:780-787.

39. Pontoglio, M., et al. 1998. Defective insulin secretion in hepatocyte nuclear factor $1 \alpha$-deficient mice. J. Clin. Invest. 101:2215-2222.

40. Johnson, J.D., et al. 2003. Increased islet apoptosis in $\mathrm{Pd} \times 1^{+/-}$mice. J. Clin. Invest. 111:1147-1160. doi:10.1172/JCI200316537.

41. Roe, M.W., Lancaster, M.E., Mertz, R.J., Worley, J.F., 3rd, and Dukes, I.D. 1993. Voltage-dependent intracellular calcium release from mouse islets stimulated by glucose. J. Biol. Chem. 268:9953-9956.

42. Johnson, J.D., and Misler, S. 2002. Nicotinic acidadenine dinucleotide phosphate-sensitive calcium stores initiate insulin signaling in human beta cells. Proc. Natl. Acad. Sci. U. S. A. 99:14566-14571.

43. Johnson, J.D., Van Goor, F., Wong, C.J., Goldberg, J.I., and Chang, J.P. 1999. Two endogenous gonadotropin-releasing hormones generate dissimilar $\mathrm{Ca}(2+)$ signals in identified goldfish gonadotropes. Gen. Comp. Endocrinol. 116:178-191.

44. Jhala, U.S., et al. 2003. cAMP promotes pancreatic beta-cell survival via CREB-mediated induction of IRS2. Genes Dev. 17:1575-1580.

45. Johnson, J.D., et al. 2004. RyR2 and calpain-10 delineate a novel apoptosis pathway in pancreatic islets. J. Biol. Chem. 279:24794-24802. 